\title{
In vivo quantification of photosensitizer fluorescence in the skin-fold observation chamber using dual-wavelength excitation and NIR imaging
}

\author{
Slávka Kaščáková • Sebastiaan de Visscher • Bastiaan Kruijt • Henriëtte S. de Bruijn • \\ Angélique van der Ploeg-van den Heuvel • Henricus J. C. M. Sterenborg • \\ Max J. H. Witjes • Arjen Amelink • Dominic J. Robinson
}

Received: 24 September 2010 / Accepted: 5 January 2011 / Published online: 29 January 2011

(C) The Author(s) 2011. This article is published with open access at Springerlink.com

\begin{abstract}
A major challenge in biomedical optics is the accurate quantification of in vivo fluorescence images. Fluorescence imaging is often used to determine the pharmacokinetics of photosensitizers used for photodynamic therapy. Often, however, this type of imaging does not take into account differences in and changes to tissue volume and optical properties of the tissue under interrogation. To address this problem, a ratiometric quantification method was developed and applied to monitor photosensitizer meso-tetra (hydroxyphenyl) chlorin (mTHPC) pharmacokinetics in the rat skin-fold observation chamber. The method employs a combination of dual-wavelength excitation and dualwavelength detection. Excitation and detection wavelengths were selected in the NIR region. One excitation wavelength was chosen to be at the Q band of MTHPC, whereas the second excitation wavelength was close to its absorption minimum. Two fluorescence emission bands were used; one at the secondary fluorescence maximum of MTHPC centered on $720 \mathrm{~nm}$, and one in a region of tissue autofluorescence. The first excitation wavelength was used to excite the MTHPC and autofluorescence and the second to excite only autofluorescence, so that this could be subtracted. Subsequently, the
\end{abstract}

S. Kaščáková • B. Kruijt • H. S. de Bruijn •

A. van der Ploeg-van den Heuvel • H. J. C. M. Sterenborg •

A. Amelink · D. J. Robinson $(\bowtie)$

Center for Optical Diagnostics and Therapy, Department

of Radiation Oncology, Erasmus Medical Center,

Room Ee-1675, PO Box 2040, 3000 CA, Rotterdam,

The Netherlands

e-mail: d.robinson@erasmusmc.nl

S. de Visscher · M. J. H. Witjes · A. Amelink

Department of Oral and Maxillofacial Surgery,

Division of Oncology, University Medical Centre Groningen,

Groningen, The Netherlands autofluorescence-corrected mTHPC image was divided by the autofluorescence signal to correct for variations in tissue optical properties. This correction algorithm in principle results in a linear relation between the corrected fluorescence and photosensitizer concentration. The limitations of the presented method and comparison with previously published and validated techniques are discussed.

Keywords Meso-tetra(hydroxyphenyl) chlorin (mTHPC Foscan $\left.{ }^{\circledR}\right) \cdot$ Ratio fluorescence imaging technique $\cdot$ mTHPC pharmacokinetics . Skin fold observation chamber

\section{Introduction}

The primary response to photodynamic therapy (PDT) is determined by the tissue oxygenation, light fluence (rate), and local concentration of the photosensitizer. The biological activity of the photosensitizer within the illuminated volume is related to the concentration of the fluorescent active form of the photosensitizer. Thus, optical imaging of photosensitizer fluorescence (using light doses much lower than are necessary for PDT damage) aids the understanding of PDT by monitoring the photosensitizer spatial distribution and its fluorescence activity.

For many years, observation chambers implanted in various animal species and in humans have been used for intravital microscopy of living tissue [1-12]. Through the possibility to transplant neoplastic tissue within the chamber, the skin-fold observation chamber was especially developed to monitor the early vascular events, anti-tumor effects, and pharmacokinetics of photosensitizers.

However, the ability to accurately quantify the in vivo measured fluorescence is critical $[13,14]$. The fluorescence 
signal originates not only from the photosensitizer but also from various other fluorescent molecules naturally present in the tissue that can cause an unknown and variable amount of background autofluorescence [15]. Moreover, the measured fluorescence signal is also influenced by geometric factors (the distance and the angle of the excitation and detection source relative to tissue surface) and the tissue optics (scattering and absorption of the excitation and fluorescence emission light in the tissue). For example, tissues with higher background absorption coefficients, e.g., due to high melanin or blood content, can decrease the propagation of both the excitation and fluorescence emission light. This is further complicated by the fact that the absorption spectrum of blood depends on its oxygenation. In addition, tumor tissue is less scattering than normal tissue [16-18] and the thickness for the different types of tissue within the chamber varies as well. In general, the tumor within the chamber is thicker than the normal tissue. Furthermore, normal tissue can also show variability in thickness at the different sites within the chamber. Since the collected fluorescence image is influenced by all of the factors discussed above (optical properties of the tissue, tissue autofluorescence, chamber thickness variations, and geometric illumination and collection factors), an imaging methodology that corrects for these factors is necessary to obtain quantitative photosensitizer fluorescence images.

Several techniques have been developed to correct the measured fluorescence for tissue autofluorescence, absorption, and scattering properties of the tissue and variations in irradiance, excitation geometry, and detection efficiency. Profio [19] calculated the ratio of fluorescence marker signal over the reference autofluorescence. Baumgartner et al. [20] and Witjes et al. [21] described a subtraction method where the autofluorescence background signal is subtracted using dual-wavelength excitation. In our group, Sinaasappel and Sterenborg developed the double ratio technique based on dual-wavelength excitation and dualwavelength detection [15] and more recently Saarnak et al. [22] published a ratiometric method based on measuring the autofluorescence signal prior to marker administration.

Bogaards et al. [14] reviewed the performance of these correction methods for various input parameters over ranges that can be expected during in vivo imaging around a standard set of optical properties representing those for human skin. The study revealed that the subtraction method of Baumgartner et al. [20] corrected the detected fluorescence signal of the marker for variations in autofluorescence, but was unable to correct for variations in irradiance, excitation geometry, and tissue optical properties. The ratiometric methods of Profio [19], Sinaasappel and Sterenborg [15], and Saarnak et al. [22] completely corrected for variations in irradiance, excitation geometry, and detection efficiency. The correction for tissue optical properties was to a great extent also achieved using ratiometric methods. However, the method of Profio [19] remained dependent on changes in autofluorescence. The method of Saarnak et al. [22] demonstrated the best quantification performance, as it depends only on the concentration of the fluorophore. This method is based on image acquisition before and after fluorophore administration, and it requires that these subsequent images be taken from the same tissue site under an identical geometry. Here the assumption was made that the optical properties and autofluorescence may change spatially within the image, but remain constant over the time interval between image acquisitions. However, these conditions are typically not met during window chamber pharmacokinetics experiments. Especially the assumption that autofluorescence, tissue optical properties, and tissue thickness remain constant over the time period for which marker pharmacokinetics is studied (typically over periods of up to 7 days) may not be valid. In this case, the method of Sinaasappel and Sterenborg [15], which does not have such restrictive conditions with respect to window chamber changes over time, was revealed to be a better option for fluorophore quantification. However, the double ratio method of Sinaasappel and Sterenborg [15] suffers from a significant drawback: the relation between marker fluorescence and marker concentration is non-linear and saturates for high marker concentrations. This severely limits the applicability of the double ratio correction technique for determining absolute fluorophore concentrations for the study of photosensitizer pharmacokinetics where large variations (several orders of magnitude) in fluorophore concentrations need to be quantified [14, 22].

In summary, two important factors limit the quantification performance of state-of-the-art ratiometric methods : (1) the remaining dependence of the methods on timedependent variations in autofluorescence, tissue optical properties, and tissue thickness [14], and (2) the relation between marker fluorescence and marker concentration is non-linear and saturates for high marker concentrations. In the present study, we address both issues using a novel ratiometric method that corrects for time-dependent variations in tissue thickness and tissue optical properties and features a linear response even for high photosensitizer concentrations. In addition, in our method, we minimize the dependence of the fluorescence signal on tissue optical properties by selecting the excitation and emission wavelengths in the red and in the near infrared (NIR), where the tissue absorption and scattering coefficients are relatively small and do not vary much with wavelength [14]. We have applied our method to measure the pharmacokinetics of the second-generation photosensitizer 5,10,15,20-tetra (m-hydroxyphenyl) chlorin (m-THPC) in the rat skin-fold observation chamber. 


\section{Experimental procedures}

Materials $\mathrm{mTHPC}\left(\right.$ Foscan $\left.^{\circledR}\right)(\mathrm{c}=4 \mathrm{mg} \mathrm{mTHPC} / \mathrm{ml}$ dissolved in PEG, EtOH, water-free solution) was obtained from Biolitec pharma (Edinburgh, The United Kingdom). Alexa Fluor 720 was obtained from Invitrogen (Breda, The Netherlands). Polyethylene glycol 400 (PEG400), Evans Blue, and Titanium dioxide $\left(\mathrm{TiO}_{2}\right)$ were obtained from Sigma-Aldrich (Zwijndrecht, The Netherlands) and 96\% ethanol (EtOH) was purchased from Merck (Amsterdam, The Netherlands).

Preparation of $m T H P C$ and administration Stock solution of mTHPC was dissolved in a solution of PEG400:EtOH: water $=3: 2: 5(\mathrm{v} / \mathrm{v})$ to a concentration $\mathrm{c}=0.126 \mathrm{mg} / \mathrm{ml}$. The solution was stored in the dark for at least $30 \mathrm{~min}$ prior to injection, after which the sample was determined to be stable, i.e., no changes in fluorescence peak intensity were detected. To investigate the state of photosensitizer after 30 min from mixing with PEG:EtOH: $\mathrm{H}_{2} \mathrm{O}$ mixture, the absorption spectra of mTHPC were recorded in the concentration range $c=0.11-200 \mu \mathrm{M}$. Over the concentration range, the spectrum of mTHPC did not change and the solution accurately followed Beer's law with no evidence of spectral peak broadening or shifts in absorption maxima. The same species of MTHPC, i.e., the monomer, is present at the concentration administrated to the animal. The prepared $\mathrm{mTHPC}$ solution was administered intravenously at a dose of $0.15 \mathrm{mg} \mathrm{kg}^{-1}$ body weight under isoflurane $/ \mathrm{O}_{2} / \mathrm{N}_{2} \mathrm{O}$ anesthesia.

Animal model The animal experiment committee of the Erasmus Medical Center approved the experimental design for this study. Skin-fold observation chambers were prepared using a slightly modified technique to the one that has been described previously [10, 23]. Briefly, the chamber was prepared on the back of female Fischer-344 rats (weight $\mathrm{m}=144 \pm 3 \mathrm{~g}$ ) in four operations (carried out under general isoflurane $/ \mathrm{O}_{2} / \mathrm{N}_{2} \mathrm{O}$ anesthesia) during the period of 2 weeks. As a result of these operations, a thin layer of subcutaneous tissue was clamped between mice and a cover slide. In the first operation, sterile air $(12 \mathrm{ml})$ was subcutaneously injected on the back of the rat to gently separate the skin from the underlying tissue. In the second operation, a plastic ring and a cover slide was positioned under the skin above the subcutaneous tissue matching the vessels. The third operation was proceeding on the second week. During this operation, the skin was folded, prepared and fixed in the splint. Finally, during the last operation, the mammary adenocarcinoma (R3230AC) tumor was transplanted in the layer of normal tissue, reached by unscrewing the cover slide on top of the chamber. Within 5-7 days, the chamber was ready for treatment, i.e., the tumor was supported by blood vessels and had visibly grown. Ideally the chamber contained fat cells and capillaries over an area of approximately $1 \mathrm{~cm}$ in diameter with some supporting arterioles and venules.

Experimental set-up for fluorescence and transmission imaging A schematic diagram of the experimental setup is presented in Fig. 1. The MTHPC fluorescence pharmacokinetics in the chamber model was investigated in time after intravenous MTHPC administration. The experimental time points were: $0 \mathrm{~min}$ (before $\mathrm{mTHPC}$ administration) and 5 min, 2 h, 4 h, 8 h, 24 h, 48 h, and $96 \mathrm{~h}$ after mTHPC i.v. injection. The localization of photosensitizer within the chamber was visualized by acquiring fluorescence and transmission images with two excitation laser light sources: a dye laser of $629 \mathrm{~nm}$ pumped by an argon ion laser (Spectra Physics, Darmstadt, Germany) and a 652-nm diode laser (Biolitec pharma, Edinburgh, The United Kingdom). Light was coupled from a bifurcated optical fiber using a system of condensing lenses into the base of a heated X-Y stage to produce a uniform distribution of both excitation wavelength lights through the sample. The fluence rate of each excitation illumination was $0.6 \mathrm{~mW} /$ $\mathrm{cm}^{2}$ and the excitation fields were uniform and equal. For fluorescence and transmission imaging, light transmitted through the chamber was collected using an $\mathrm{f} 2,8 / 105 \mathrm{~mm}$ macro lens and imaged onto a CCD camera (ORCA-ER, Hamamatsu, Japan). The macro lens can be used to zoom in on a specific area within the sample resulting in a square field of view of $4.5 \mathrm{~mm}$. Detection filters were placed in a filter wheel (L.O.T.-Oriel, Stratford, USA) between the macro lens and the CCD camera in order to obtain the fluorescence (band-pass filter $720 \pm 10 \mathrm{~nm}$ (Omega Optical, Blattleboro, USA), long-pass filter $763 \mathrm{~nm}$ (transmission: 763-1,050 nm, Omega Optical, Blattleboro, USA)) and transmission (neutral density filter with $10 \%$ transmission for excitation wavelength light sources $652 \mathrm{~nm}$ and $629 \mathrm{~nm}$, Omega Optical, Blattleboro, USA). To eliminate

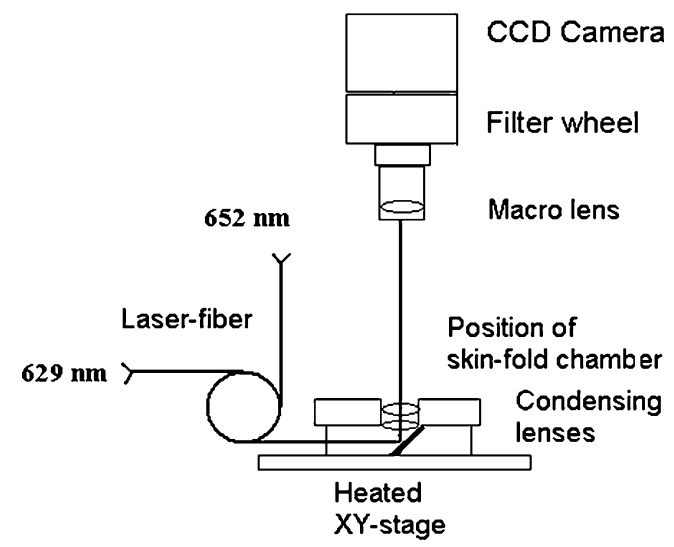

Fig. 1 The schematic diagram of the experimental setup 
the presence of excitation light in the fluorescence detection channels, the identical long-pass filters of $690 \mathrm{~nm}$ (transmission: 690-1,050 nm, Omega Optical, Blattleboro, USA), were placed together with band-pass filter $720 \pm 10 \mathrm{~nm}$ and long-pass filter $763 \mathrm{~nm}$. In addition, to shield the CCD camera from very long wavelength light, a short-pass filter of $850 \mathrm{~nm}$ (transmission: $845-585 \mathrm{~nm}$, blocking: $880-1,100 \mathrm{~nm}$, L.O.T.Oriel, Stratford, USA) was placed between the exit of the filter wheel and the CCD camera. The integration time of the camera was $30 \mathrm{~s}$ for each excitation and a filter combination was used to visualize tissue fluorescence. The fluence delivered during these measurements was approximately $0.072 \mathrm{~J} \mathrm{~cm}^{-2}$ per measurement time point. The total fluence delivered to each chamber was approximately $0.504 \mathrm{~J} \mathrm{~cm}^{-2}$ during the course of the experiment. Between measured time points, animals were conscious and placed in a dark and warm environment.

Principle of correction method The ratiometric method used in this study is illustrated in Fig. 2, where the in vivo absorption and emission spectra of mTHPC are presented with the combined transmission characteristics of the filters used for fluorescence detection.

The method presented here is based on dual-wavelength excitation and dual-wavelength detection: One excitation

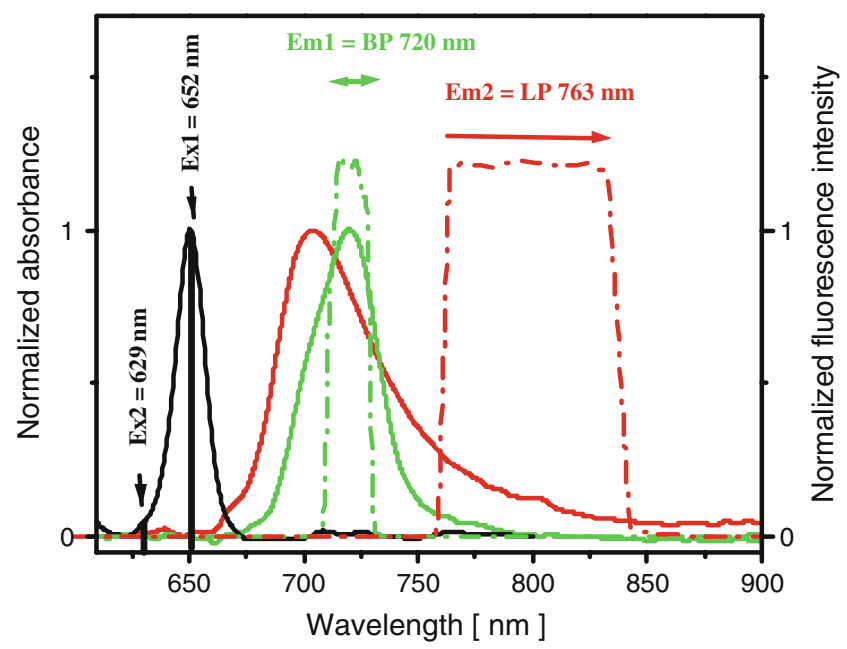

Fig. 2 Schematic excitation (black line) and emission spectra (excitation wavelength $652 \mathrm{~nm}$ ) (green line) of mTHPC based on in vivo measurement from rat muscle tissue $16 \mathrm{~h}$ from i.v. injection of mTHPC $\left(\mathrm{c}=0.15 \mathrm{mg} \cdot \mathrm{kg}^{-1}\right)$. The red line shows the tissue autofluorescence (excitation wavelength $652 \mathrm{~nm}$ ) measured in vivo from rat muscle tissue. Presented spectra are based on previous measurements monitoring mTHPC in muscle tissue using differential path length spectroscopy (DPS) and fluorescence differential path length spectroscopy (FDPS) [32]. The figure also includes transmission characteristics of band-pass filter $720 \pm 10 \mathrm{~nm}$ (dash dot green line) and longpass filter $763 \mathrm{~nm}$ (dash-dot red line) in combination with short-wave pass filter $850 \mathrm{~nm}$ (used to shield the CCD camera from very long wavelength light) wavelength is chosen to be at an absorption maximum of mTHPC and the other at its absorption minimum. The two emission wavelengths are chosen to be at the secondary fluorescence maximum of mTHPC (at $\lambda=720 \mathrm{~nm}$ ) and in the region of no photosensitizer fluorescence. The reason for such a wavelength selection was to detect the changes in the amount of mTHPC fluorescence and monitor the autofluorescence changes in real time. The excitation of mTHPC with 652-nm wavelength (Q-band absorption maximum of mTHPC (Fig. 2)) will produce the highest fluorescence compared to excitation by any other wavelength in the red region of the MTHPC spectra. The second excitation wavelength was $629 \mathrm{~nm}$. This excitation wavelength corresponds to an absorption minimum of mTHPC (Fig. 2). Therefore, excitation of mTHPC with 629-nm wavelength leads to only small amounts of mTHPC fluorescence emission at $720 \mathrm{~nm}$. Furthermore, the fluorescence of mTHPC decreases rapidly beyond $730 \mathrm{~nm}$ (with a minimal fluorescence for wavelengths above 750). Therefore, excitation at $629 \mathrm{~nm}$ and detection of fluorescence for wavelengths longer than $763 \mathrm{~nm}$ will give us information on how background autofluorescence changes with time. It is important to note that fluorescence emission detected in the band-pass filter $720 \pm 10 \mathrm{~nm}\left(\lambda_{\text {exc }}=652 \mathrm{~nm}\right)$ is a spectral convolution of mTHPC fluorescence and background autofluorescence. Therefore, correction for background autofluorescence in the band-pass filter $720 \pm 10 \mathrm{~nm}$ is necessary. For this purpose, the subtraction of fluorescence signal detected in the band pass filter $720 \pm 10 \mathrm{~nm}$ by excitation at $629 \mathrm{~nm}$ was included. Subsequently, to ensure correction for tissue optical properties, this difference is divided by the autofluorescence signal excited by $629-\mathrm{nm}$ wavelength and detected in the region of wavelengths longer than $763 \mathrm{~nm}$.

Two assumptions are made: (1) the excitation at $629-\mathrm{nm}$ and 652-nm wavelengths lead to the same autofluorescence, i.e., difference in yield of fluorescence for the tissue fluorescence by excitation at $652 \mathrm{~nm}$ and $629 \mathrm{~nm}$ is minimal; (2) the excitation by $629-\mathrm{nm}$ wavelength does not lead to mTHPC fluorescence detection at wavelengths longer than $763 \mathrm{~nm}$.

Subtraction and division of the images was performed according to the following formula:

$R=\frac{F\left(\lambda_{\text {exc652nm }}, \lambda_{\text {emisBP720nm }}\right)-F\left(\lambda_{\text {exc629nm }}, \lambda_{\text {emisBP720nm }}\right)}{F\left(\lambda_{\text {exc629nm }}, \lambda_{\text {emisLP763nm }}\right)}$,

where $F\left(\lambda_{\text {exc }} 652 \mathrm{~nm}, \lambda_{\text {emis BP }} 720 \mathrm{~nm}\right)$ is the fluorescence image detected by excitation light source of $652 \mathrm{~nm}$ in the wavelength region $720 \pm 10 \mathrm{~nm} ; \mathrm{F}\left(\lambda_{\text {exc }} 629 \mathrm{~nm}, \lambda_{\text {emis BP }} 720 \mathrm{~nm}\right)$ is fluorescence image registered by excitation light of $629-\mathrm{nm}$ wavelength and fluorescence detected in the wave- 
length region $720 \pm 10 \mathrm{~nm}$ and $\mathrm{F}\left(\lambda_{\text {exc } 629 \mathrm{~nm},} \lambda_{\text {emis LP } 763 \mathrm{~nm}}\right)$ is the image excited by wavelength $629 \mathrm{~nm}$, fluorescence detection for the wavelength region $>763 \mathrm{~nm}$.

Image analysis Image analysis was performed using the Labview 7.1 (National Instruments Corporation, Austin, USA). Images were first corrected for background and minor variations in fluence rate of the corresponding excitation light sources. In the second step, the sequence of fluorescence images from each animal was registered by translation and rotation using anatomical landmarks identified in the corresponding transmission images. In the next step, binning of the pixels was performed $(4 \times 4)$ to increase the signal-to-noise ratio. After the pixel binning, the images were resized on the same size of 16-bit image at a resolution of $1344 \times 1024$ pixels. The registration of images from the second step of analysis enabled us to determine the fluorescence intensity of each tissue type from the same area as follows: in the corresponding transmission image the regions of interest were chosen for each tissue type. Tumor and normal tissue regions of interest were chosen so that no large vessels were in, or close to, the region. Thus in every animal, three regions of interest were chosen for normal tissue, three regions of interest selected inside the tumor, and, depending on the vessel's content, three up to five regions of interest were selected within the vessels. The same selected regions of interest were also applied for images corrected by ratio imaging technique, where the subtraction and division of the images was done according to Eq. (1).

To investigate the validity of our ratiometric imaging correction technique, we analyzed vessels of various diameters. Blood vessel diameter was estimated from the transmission images. According to determined square field of view of macro lens, one picture element (pixel) corresponds to approximately $3 \mu \mathrm{m}$.

Statistics In the present study, three female Fischer-344 rats were used to determine autofluorescence and transmission-intensity profiles. However, the mTHPC pharmacokinetics profile was evaluated just in one animal. The aim of our current study is to present the correction method and not to validate the mTHPC pharmacokinetics profile. Presentation of the results from one animal avoids the increase in standard deviations due to intra-animal (biological) variations.

Student's $t$ test was used to determine significance for the difference in autofluorescence signal measured by two different excitation light sources. Results with a $p$ value below 0.05 were considered significant. Statistical analysis was done using Microcal Origin, version 6.0 (Microcal Software, Inc., Northampton, MA).

\section{Results}

Window chamber tissue optical properties and autofluorescence

Figure 3 shows the white light images of the chamber model immediately before mTHPC injection and $96 \mathrm{~h}$ later. Tumor tissue is easily recognized as a circular area of higher light transmission compared to the surrounding normal tissue. Vessels can be recognized as visually darker tissue, i.e., more light-absorbing areas. In comparison with the first day of the experiment (Fig. 3a), after 96 h (Fig. 3b), the natural changes in the chamber model are clearly visible. The tumor size increased and the position of the vessels surrounding the tumor changed.

Figure 4 demonstrates the transmission intensity time profile of 652-nm excitation light collected from different tissue types within the transmission images of the chamber. In comparison with the vessels and normal tissue, the transmission intensity of the light is highest for the tumor tissue. The lowest light transmission is detected from the vessel's area. The difference is visible for all observation time points. Within the time, no significant changes in the
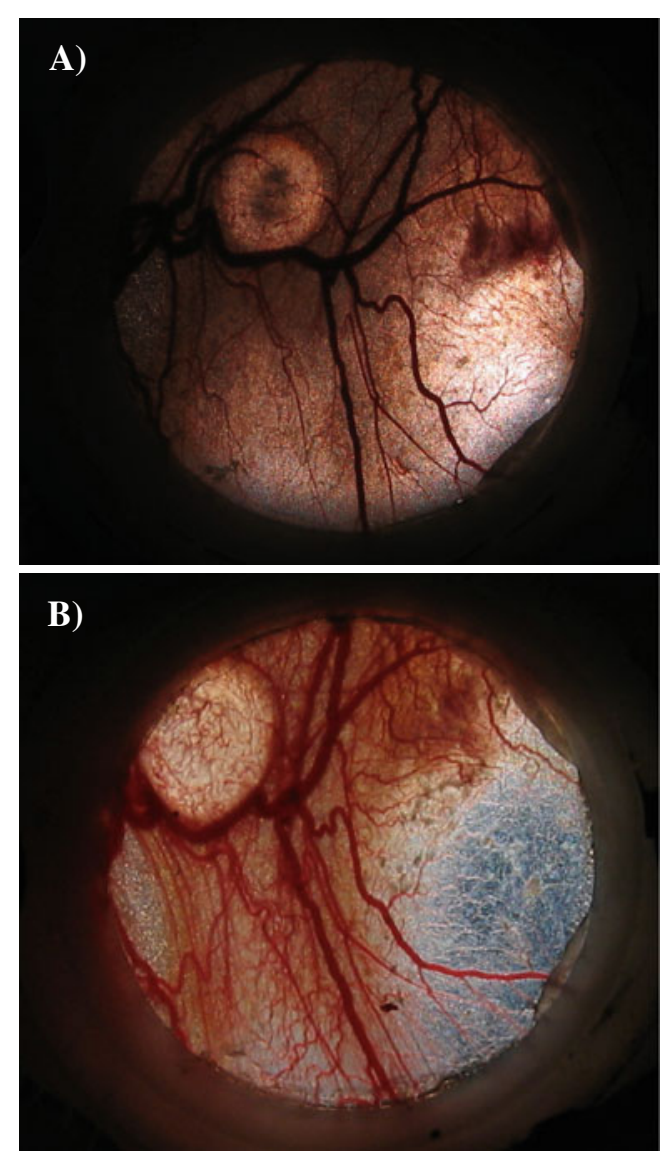

Fig. 3 White light reflection images of a chamber acquired (a) before and (b) $96 \mathrm{~h}$ after mTHPC adminisration 


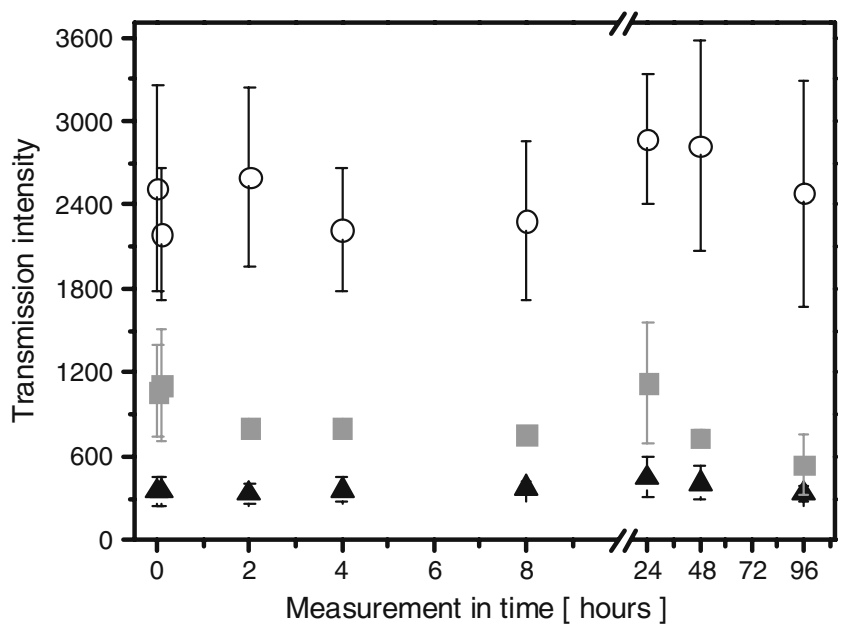

Fig. 4 Transmission-intensity time profile of $652-\mathrm{nm}$ excitation light collected from different tissue types : ( $\boldsymbol{\Delta})$ vessels, $(\square)$ normal tissue, and (०) tumor area. The standard deviation for time point within the tissue type was calculated from region of interests of three control animals (in every animal, three up to five regions of interest were selected for tissue type

transmission profile of 652-nm excitation light are detected in any of the tissue types, i.e., the intensity of the transmission light does not change in time.

In Fig. 5, the difference between fluorescence intensities of the background autofluorescence (measured before mTHPC administration) excited by wavelengths $629 \mathrm{~nm}$ and $652 \mathrm{~nm}$, detected in the emission channels (band-pass $720 \pm 10 \mathrm{~nm}$ and long-pass $763 \mathrm{~nm}$ ) is presented. For both detection channels, for the same tissue type, the fluorescence intensity using 652-nm excitation was not significantly different $(p>0.05)$ from the 629-nm excitation.

Figure 6 shows the autofluorescence kinetics (excitation at $629 \mathrm{~nm}$, long-pass filter $763 \mathrm{~nm}$ ). The fluorescence kinetics for all tissues changed in the same manner, i.e., from the measurement time points beyond $24 \mathrm{~h}$, an increase in fluorescence intensity is detected. The autofluorescence signal from the vasculature is 1.2 times lower than for normal tissue and 1.5 times lower in comparison with fluorescence intensity detected in the tumor area.

\section{Fluorescence pharmacokinetics of mTHPC}

In Fig. 7, the time profile of the chamber model after mTHPC administration is demonstrated. Figure 7 a shows the time profile of chamber transmission images. The time-dependent evolution of the uncorrected fluorescence images (excitation $652 \mathrm{~nm}$, detection BP 720) of mTHPC pharmacokinetics within the chamber model is shown in Fig. 7b. In all uncorrected fluorescence images, the borders of the chamber are clearly visible due to the fluorescence properties of the plastic ring surrounding the chamber.
A)

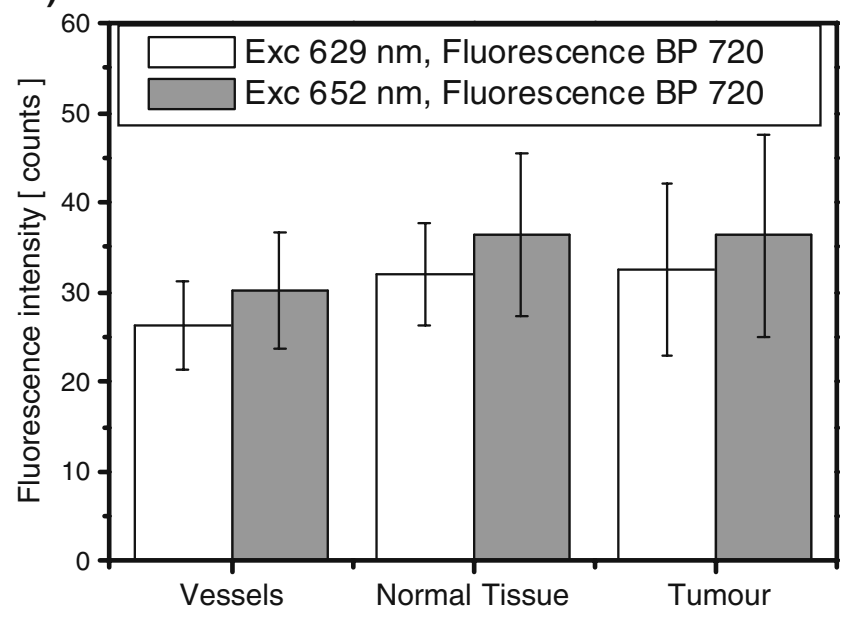

B)

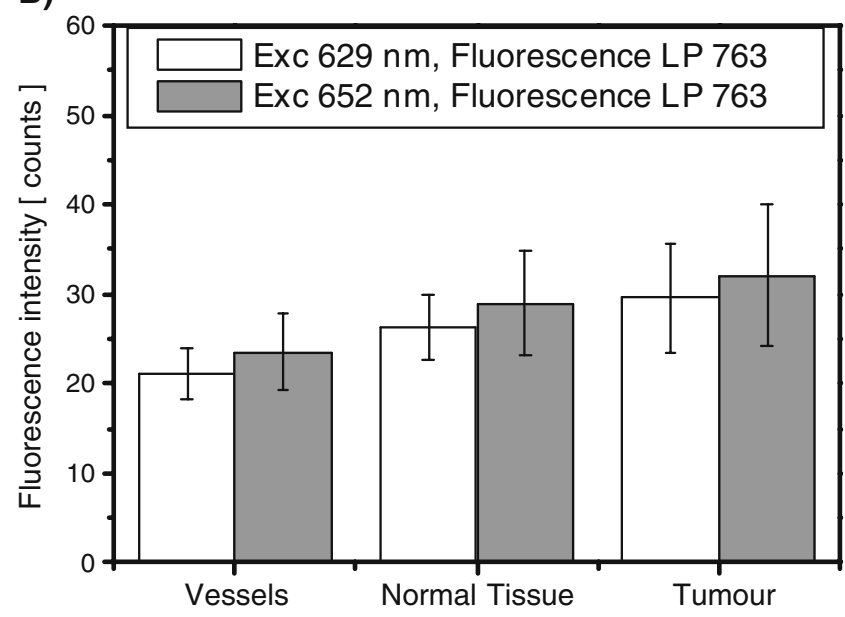

Fig. 5 Fluorescence intensity collected from different tissue types in control animals (without mTHPC, corresponding to the time $0 \mathrm{~h}$ from the chamber preparation) using a band pass filter $720 \pm 10 \mathrm{~nm}$, b longpass filter $763 \mathrm{~nm}$. White and gray columns represent $629-\mathrm{nm}$ and 652-nm excitation, respectively. The standard deviation was calculated from region of interests of the same tissue type of three control animals (in every animal, three up to five regions of interest were selected for tissue type)

Figure $7 \mathrm{c}$ shows the mTHPC uncorrected fluorescence pharmacokinetic profile within the different tissue types. Five minutes after mTHPC administration, the fluorescence intensity is highest in the vessels and decreases for longer time points. Four hours after mTHPC administration the difference of fluorescence observed in vessels and other tissue types reduces and from time points beyond $8 \mathrm{~h}$ after mTHPC administration the fluorescence distribution in tumor and normal tissue is similar. Normal and tumor tissue showed no significant difference in fluorescence intensity over the investigated period and follow the same type of kinetic profile (Fig. 7c): between 5 min and $8 \mathrm{~h}$, the fluorescence intensity does not change significantly, while above the 8 -h time point, an increase in fluorescence is 


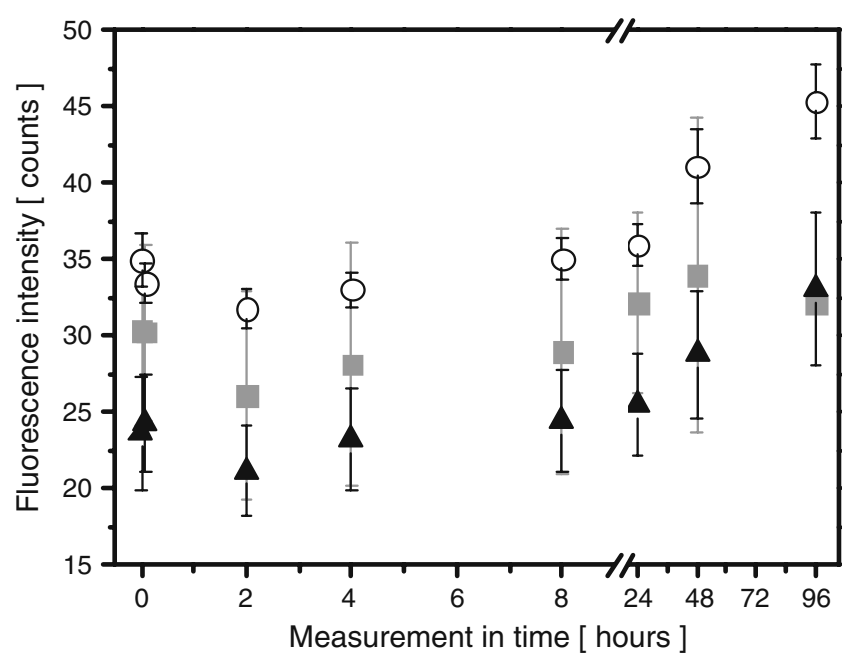

Fig. 6 Fluorescence pharmacokinetics of autofluorescence (629-nm excitation, > 763-nm detection) for different tissue types: $(\boldsymbol{\Delta})$ vessels, (I) normal tissue and ( $\circ$ ) tumor area. The standard deviations represent variations between minimum three regions of interest for each tissue type within the chamber model of one animal

observed. The intensity increased up to $48 \mathrm{~h}$ after mTHPC administration and decreased for the 96-h time point. For all incubation time points, the standard deviations are higher for normal tissue than for tumor tissue or vessel area.

The time-dependent evolution of the corrected fluorescence images of mTHPC (images corrected by ratio imaging technique, formula [1]) within the chamber is shown in Fig. 7d. According to transmission images (Fig. 7a), at time point $5 \mathrm{~min}$ from mTHPC administration, only vessels show fluorescence. The intensity of fluorescence within the vessels decreased for longer incubation time points. At $t=4 \mathrm{~h}$ the contours of the vessels are not as clear anymore due to mTHPC penetration through vessel walls into the tissue. For $t>8 \mathrm{~h}$ after mTHPC administration the fluorescence is now within all structures of the chamber. Note that the fluorescence of the plastic ring (Fig. 7b) is not visible in any of corrected images (Fig. 7e).

The time dependence of the corrected fluorescence intensity for the different tissue types is plotted in Fig. 7e. The signal detected from the vessels shows high variations (large error bars) for times below $24 \mathrm{~h}$. The variation was highest for $5 \mathrm{~min}$ followed by decreasing variations for longer incubation time points. Beyond $24 \mathrm{~h}$, these variations minimized. In contrast, variations of the corrected fluorescence signal for normal tissue and tumor tissue are small throughout the time-course of the experiment.

\section{Vessel diameters}

Figure 8 shows the corrected fluorescence signal as a function of time from mTHPC administration detected in vessels of different diameters. The pharmacokinetic profile of mTHPC fluorescence is similar for all vessels: $5 \mathrm{~min}$ after mTHPC administration, the fluorescence intensity appears to be the highest, followed by a decrease for longer time points. Between 24 and $96 \mathrm{~h}$, no significant changes of fluorescence intensity are detected. However, the corrected fluorescence signal in Fig. 8 demonstrates large differences in fluorescence intensity for the different vessel diameters. With decreasing vessel diameter, the fluorescence intensity decreases. The difference of fluorescence intensity is highest for early time points after mTHPC i.v. administration $(t<24 \mathrm{~h})$ : the highest difference is detected for the 5 min time point followed by a decrease in difference up to $8 \mathrm{~h}$. Between the 24 and 96-h time points, the fluorescence intensity does not show any dependence on vessel diameter.

\section{Discussion}

We have investigated the use of a ratiometric imaging technique for monitoring the kinetics of mTHPC fluorescence in the rat skin-fold observation chamber. The chamber model was specifically designed for monitoring the pharmacokinetics of photosensitizers used in PDT. However, a problem associated with fluorescence measurements is the difficulty of obtaining quantitative fluorophore fluorescence, due to varying optical properties of tissues and differences in tissue thickness. This has important consequences for the general interpretation of fluorescence measurements in the window chamber. For example, in a previous study, our group determined the spatial distribution of the kinetics of protoporphyrin IX (PpIX) fluorescence during ALA-PDT [11]. PpIX fluorescence kinetics were measured in different tissue types and conclusions were based on data that was not corrected for differences in tissue optical properties and differences in the thickness within and between window chambers. Although the conclusions were based on determining the rate of fluorescence increase in each tissue type separately, temporal variations in tissue optical properties and differences in the thickness of different tissues were not considered. This may be of particular importance, especially when the relationship between the increase of PpIX fluorescence with distance from an arteriole and venule was investigated. Thus in the present study, our intention was to investigate the difference in optical properties of different types of tissue and its time changes in the chamber model. The methodology proposed here accounts for the wavelength dependence of tissue optical properties and overcomes the non-linearity of previously published ratiometric methods.

Tissue optical properties of chamber

Figures 4, 5, 6 clearly show the differences between the fluorescence and transmission intensity acquired from 
Fig. 7 The time profile of the chamber model (the same window chamber as one analyzed for Fig. 6) after mTHPC administration. a Time profile of chamber transmission images after MTHPC administration. b Time profile of uncorrected fluorescence emission images (652-nm excitation, $720 \pm 10-\mathrm{nm}$ detection) of a chamber after mTHPC administration. c The time-dependent evolution of the corrected fluorescence images of chamber model after mTHPC administration. d mTHPC pharmacokinetics profile (uncorrected fluorescence signal: $652-\mathrm{nm}$ excitation, $720 \pm 10-\mathrm{nm}$ detection) for different tissue types: ( $\mathbf{\Delta}$ ) vessels, ( $\square$ ) normal tissue and (o) tumor area. e mTHPC

pharmacokinetics profile (corrected fluorescence signal) within the different tissue types: $(\Delta)$ vessels, ( ) normal tissue and $(\mathrm{O})$ tumor area
A)
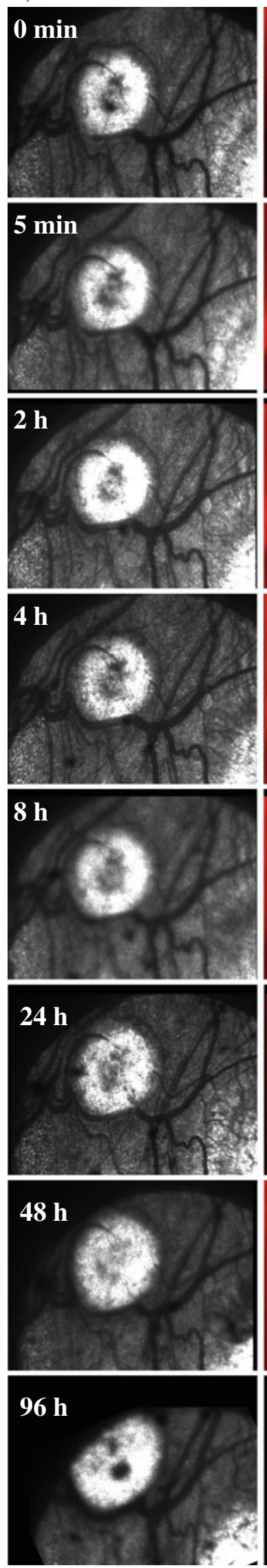

B)

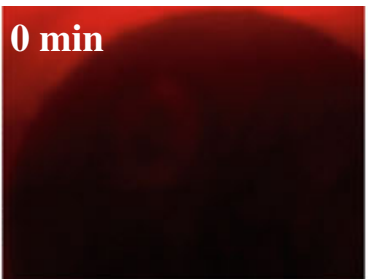

$5 \mathrm{~min}$

$5 \mathrm{~min}$

\section{$2 \mathrm{~h}$}

$2 \mathrm{~h}$

o min
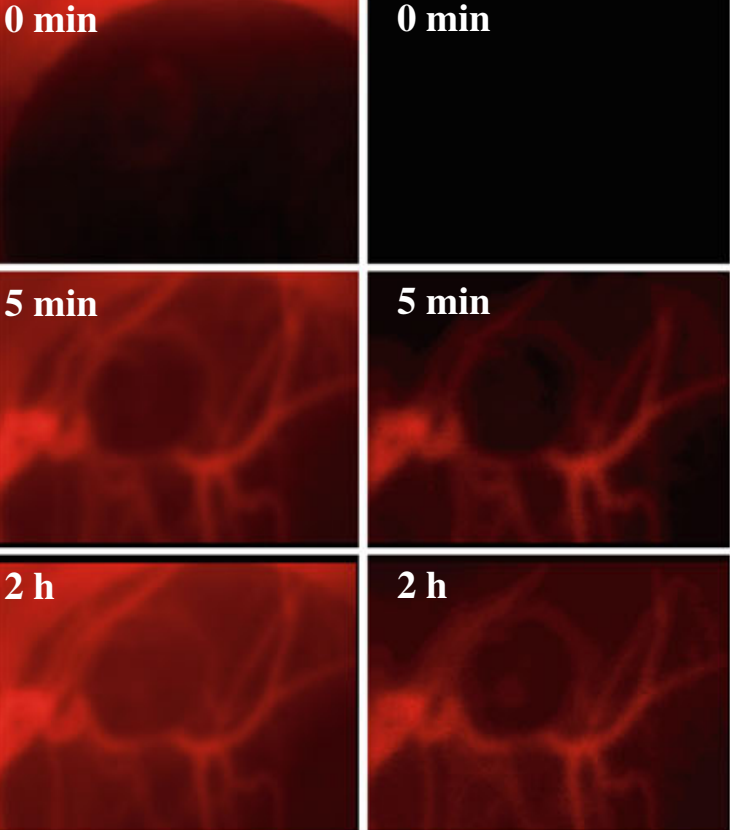

$4 \mathrm{~h}$

$4 \mathrm{~h}$

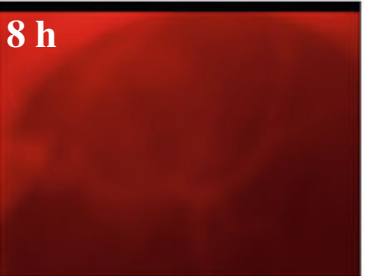

8 h

$24 \mathrm{~h}$

$24 \mathrm{~h}$

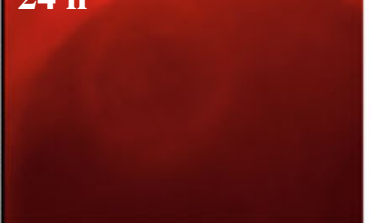

\section{$48 \mathrm{~h}$}

$48 \mathrm{~h}$
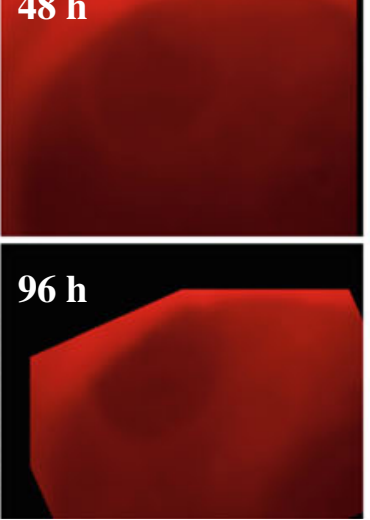

\section{$96 \mathrm{~h}$}




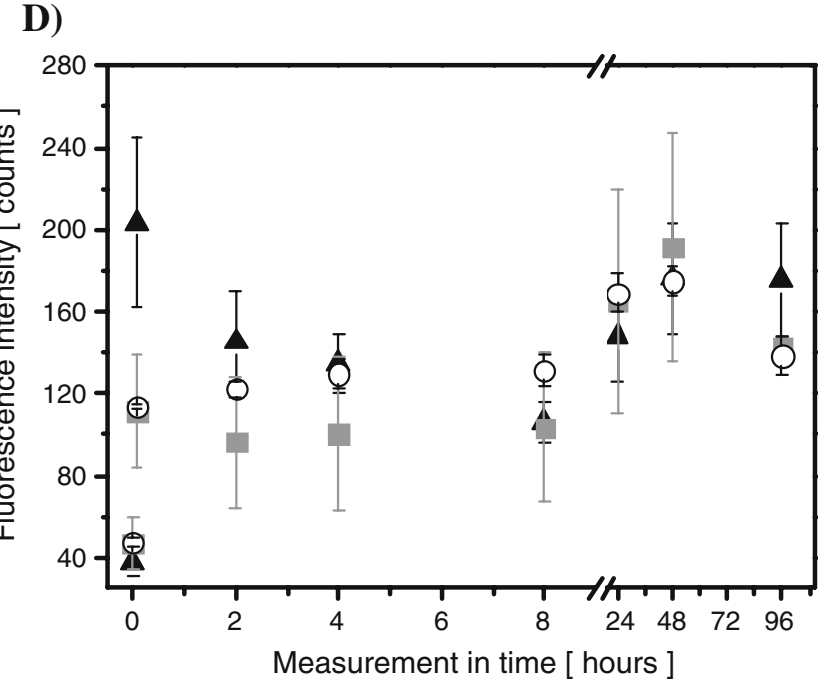

E)

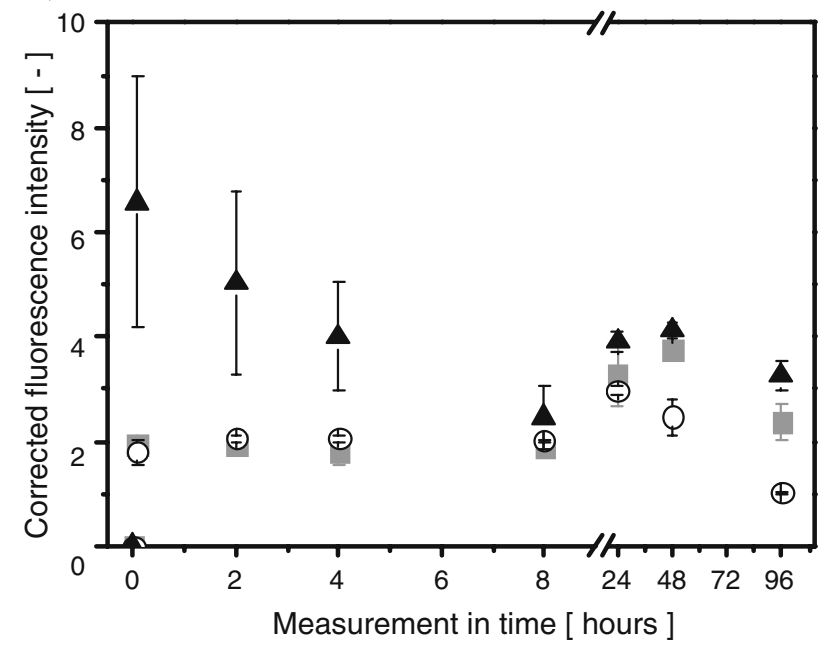

Fig. 7 (continued)

different types of tissue during the course of the experiment. The fluorescence intensity detected from vessels was lower than that from normal or tumor tissue. Considering the blood content within these vessels and the absorption spectra of oxy- and deoxy hemoglobin, the result observed in Fig. 5a or b is not surprising. The same effect is also evident in the transmission-intensity profile from different tissue types (Fig. 4), and in the transmission images of the chamber (Fig. 7a). The chamber vasculature represents the tissue with the highest absorption coefficient, which significantly attenuates the propagation of light. In contrast, tumor tissue exhibits higher autofluorescence intensity than the normal tissue area (Figs. 5, 6). This might be caused by (1) a lower absorption coefficient of tumor tissue; (2) a different scattering coefficient of tumour tissue (it is not evident whether a higher or lower scattering coefficient would result in a higher fluorescence yield); (3) a larger thickness of tumour tissue; (4) a higher native fluorophore

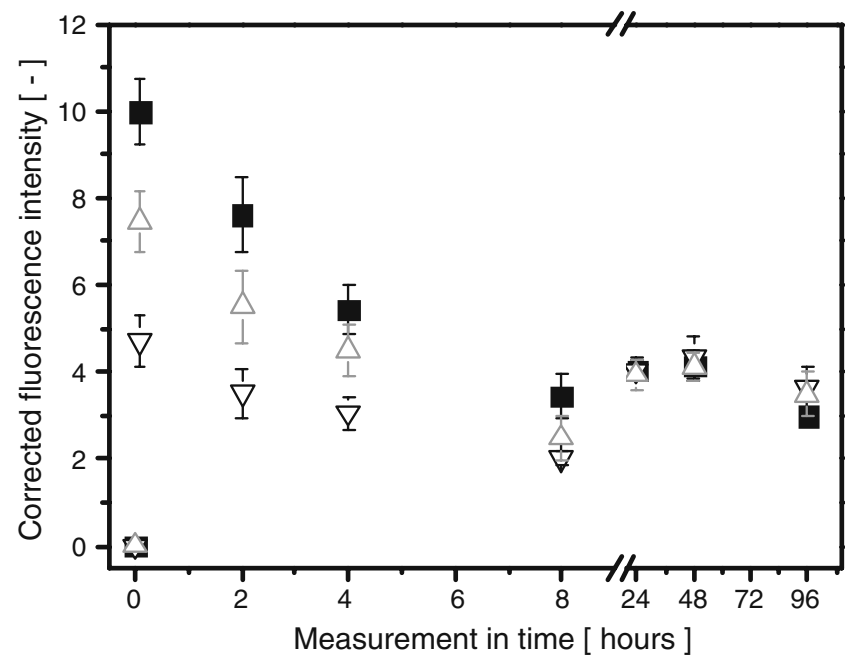

Fig. 8 Corrected fluorescence signal as a function of the time from mTHPC administration, detected in the vessels of different diameter: (घ) $104 \mu \mathrm{m},\left({ }^{\Delta}\right) 83 \mu \mathrm{m}$ and $(\nabla) 47 \mu \mathrm{m}$

concentration in the tumor tissue. Most likely, a combination of these factors results in the observed difference. Since the raw fluorescence signal is confounded by each of these factors, the differences in (and changes to) optical properties of the tissue under interrogation will lead to significant quantification errors of photosensitizer fluorescence within the chamber. Clearly a method that corrects for the influence of these effects would be a step forward.

\section{Validation of assumptions}

In the study of Bogaards et al. [24], it was shown that the performance of imaging techniques can be improved by selecting the excitation and emission wavelengths towards the NIR. Thus, in this study, the excitation wavelengths were carefully selected between 620 and $840 \mathrm{~nm}$, where the tissue absorption and scattering are relatively small. In addition, the combination of exciting tissue fluorescence at a wavelength where the photosensitizer absorbs minimally and detection at the wavelength of no photosensitizer fluorescence, allowed us to monitor kinetics of tissue autofluorescence during the course of the experiment. According to the principle of the method we have presented (Eq. 1), the assumption was made that both excitation wavelengths used in this study lead to the same autofluorescence. This assumption is confirmed in Fig. 5, where the fluorescence intensity detected from different tissue types of control animals is plotted. Within the same tissue type, the fluorescence intensity was not significantly different using 629- and 652-nm excitation.

The second assumption underlying our correction algorithm is that only signals from tissue autofluorescence can be detected in the wavelength region $>763 \mathrm{~nm}$, using 629 $\mathrm{nm}$ wavelength excitation. This choice of wavelengths was 
based on the minimal absorption of mTHPC at the excitation wavelength and on the lack of MTHPC emission in the wavelength region $>763 \mathrm{~nm}$ (Fig. 2). In Fig. 6, we did not detect any changes of autofluorescence within the first measured time points in any of the tissue types. In contrast, mTHPC fluorescence pharmacokinetics profile (excitation $652 \mathrm{~nm}$, fluorescence detection at $720 \pm 10 \mathrm{~nm}$; Fig. 7c), revealed large differences in fluorescence intensity within the vessels $5 \mathrm{~min}$ after the mTHPC administration. It is very unlikely that there are changes of tissue autofluorescence over the timescale of these first few measurements compared to the first measurement of autofluorescence (at $t=0$ ). Therefore, we conclude that the increase of fluorescence intensity observed in chamber vessels in the band-pass filter $720 \pm 10 \mathrm{~nm}$ (Fig. 7b, c) is due to fluorescence emission of mTHPC. The fact that this effect was not seen in Fig. 6 confirmed that the signal detected in the wavelength region $>$ $763 \mathrm{~nm}$ (using 629-nm wavelength excitation) is solely due to tissue autofluorescence.

Temporal changes of tissue optical properties within the chamber

The detection of autofluorescence within the timeframe of the experiment (Fig. 6) revealed that the autofluorescence kinetics for all tissues changed in the same manner, i.e., for measurement time points above $24 \mathrm{~h}$ there is a (steady) increase in autofluorescence intensity. The cause of the observed effect might be chamber thickness changes and/or the changes in tissue optical properties at later time points. Unfortunately, our data does not present conclusive evidence as to which of these effects is dominant. The fact that these changes occur within the time-course of the experiment demonstrates the necessity for a correction method, since the marker fluorescence will be affected by these chamber changes similar to the autofluorescence.

\section{Fluorescence pharmacokinetics of mTHPC}

Figures 4, 5, and 6 clearly illustrate the necessity of an appropriate correction technique for quantitative measurement of photosensitizer fluorescence in the window chamber. If we were to base conclusions on the pharmacokinetics profile of mTHPC from Fig. 7c (uncorrected fluorescence profile), then the tumor/normal tissue ratio is significantly higher at early time points after mTHPC administration. Taking into account Figs. 4, 5, and 6, this is actually due to a larger thickness and/or smaller absorption/scattering content of tumor tissue, rather than due to a higher mTHPC concentration within the tumor. The larger standard deviation associated with data detected from normal tissue can be explained by thickness inhomogeneities and due to the presence or absence of micro-capillaries. These variations are only visible at high magnification and are difficult to avoid in a model where normal tissue has a significant component of microvasculature.

The correction of raw fluorescence data using the ratiometric correction method that we presented results in a significant decrease in the spatial variation associated with a single measurement time point (evidenced by smaller error bars) and does not report a significant difference between the profile of MTHPC pharmacokinetics between tumor and normal tissue up to $24 \mathrm{~h}$ after the administration of mTHPC (Fig. 7e). In addition, we observe that in all uncorrected images, a fluorescent border to the chamber is clearly visible (Fig. 7b). This is due to the presence of a fluorescent plastic ring on which the chamber is mounted. Corrected fluorescence images do not show this artifact (Fig. 7d).

\section{Limitations of the correction method}

As expected, the uncorrected signals (Fig. 7c) showed larger intra-chamber variations (visible as error bars) than the signal corrected using the ratiometric technique (Fig. 7e). The exception to this trend was observed in vessels where the error bars were still high at early time points. These large error bars for mTHPC in vessels at early time points are caused by averaging contributions from small and large vessels, combined with vessel-diameterdependent mTHPC fluorescence yields for early time points. The dependence of corrected MTHPC fluorescence pharmacokinetics profile as a function of vessel diameter (Fig. 8) shows that for larger diameter vessels we obtained a higher fluorescence intensity of MTHPC than for small vessels. We believe that the origin of this effect is due to a limitation of our correction method, which is illustrated by considering the spatial distribution of absorbers and fluorophores within the vasculature at early time points (Fig. 9). While it is well known that there are a number of circulating endogenous fluorophores within the vessels, such as water-soluble porphyrins and erythrocytes themselves [25-27], the autofluorescence from the window chamber vasculature is likely to be dominated by the contribution from connective tissue of vessel walls (in particular the tunica media) and the surrounding tissue. For early time points, mTHPC is primarily localized in the blood plasma [28-31]. Thus localization of mTHPC is inhomogeneous with respect to the tissue background autofluorescence (Fig. 9b). Since our ratiometric method corrects for tissue optical properties and chamber thickness using the background autofluorescence, our method is most suitable for situations where the marker fluorescence and autofluorescence are co-localized; a condition that is not met in case of mTHPC at early time points. A confirmation of our hypothesis is the absence of a dependence of 


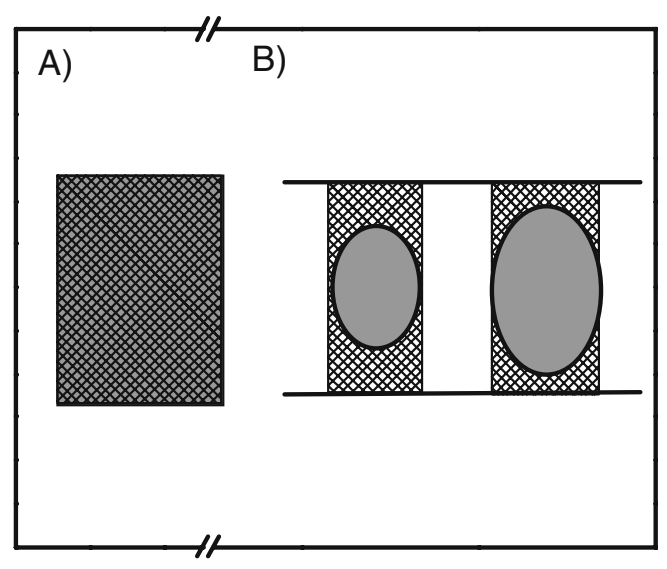

Fig. 9 Schematic representation of a limitation of the correction technique a The same distribution pattern of fluorescence signal for fluorophore (filled gray area) and background tissue (hashed area). b Vertical profile of tissue with vessels of different diameter. Vessel is shown as an ellipse within the tissue. Localization of fluorophore (gray) is inhomogeneous compared to the tissue background fluorescence (open hashed area)

mTHPC fluorescence on vessel diameter for later time points (between 24 and $96 \mathrm{~h}$ ). Here the progressive redistribution of mTHPC from the plasma into and through the vasculature into normal tissue results in a correction that is not influenced by the different spatial distribution of autofluorescence and marker fluorophore. This redistribution of MTHPC is in accordance with the previously published pharmacokinetic profile of mTHPC in blood and other tissues [28-31]. Plasma mTHPC levels are high immediately after mTHPC injection $(5 \mathrm{~min}$ after i.v. administration) and decrease exponentially thereafter. In mice and rats, mTHPC shows a bi-exponential decline with half-life values of $0.5-1.3 \mathrm{~h}$ for the initial decline and 6.9$20.9 \mathrm{~h}$ for the elimination phase [28-31].

Optical phantoms and double ratio imaging

We tested the performance of our correction algorithm in optical phantoms. To maintain the geometry of our transmission measurements and to avoid the precipitation of scattering centers, we prepared solid silicone phantoms containing mTHPC. We chose to use the absorber Evans Blue and $\mathrm{TiO}_{2}$ to simulate tissue absorption and scattering. Preparing solid phantoms with spectral properties that match those of MTHPC and tissue autofluorescence in vivo proved to be very challenging. Unfavorable spectral shifts and changes in extinction coefficients and fluorescence quantum yield were observed in dyes we selected to match the optical properties of tissue. For example, the choice of phantom autofluorophore was particular difficult. We found that in silicone Alexa-Fluor 720 had a negligible fluorescence quantum yield whereas Evans Blue showed significant fluorescence at both excitiation wavelengths. Given these problems, we chose to compare our correction algorithm with a previously validated ratiometric technique, in which a double ratio (DR) is formed using two excitation and two detection wavelengths [15]. The result of this analysis can be seen in Fig. 10, where the mTHPC pharmacokinetic profile within the different tissue types is presented. A comparison of the present correction method (Fig. 7e) shows a similar pharmacokinetics profile for all tissue types. However, there is an important difference between DR profiles in areas of very high fluorescence intensity, in particular for short administration times in vessels. The non-linear relationship between double ratio and fluorophore concentration is a well-understood effect of double ratio imaging [14]. This saturation effect is a significant limitation of the DR correction technique for determining absolute fluorophore concentrations that are necessary for the study of pharmacokinetics, as discussed previously.

It is clear that the method we present as well as the DR correction technique have important limitations [14] and it is important to stress that the choice of the fluorescence detection technique and correction method should be based on a specific application. This is particularly true for the most appropriate selection of excitation wavelengths and detection wavelength bands. It is important to note that we have applied our correction method in a study investigating mTHPC detection. The application of this technique to different photosensitizers (or other fluorescent species) should involve the careful consideration of the spectroscopic properties of the photosensitizer, so that autofluorescence can be measured during the course of the study in real time and the method remains linearly dependent on photosensitizer concentration.

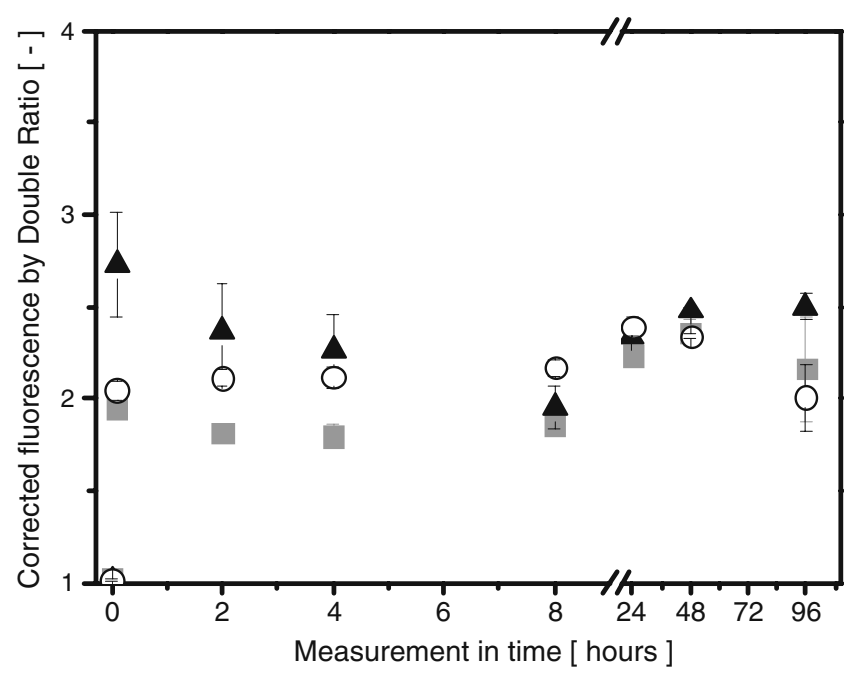

Fig. 10 mTHPC pharmacokinetics profile (corrected fluorescence signal by double ratio fluorescence imaging technique of Sinaasappel and Sterenborg [15]) within the different tissue types: $(\boldsymbol{\Delta})$ vessels, $(\mathrm{C})$ normal tissue and $(\circ)$ tumor area 


\section{Conclusions}

In summary, we have shown that the raw fluorescence intensity collected from tissues in the skin-fold observation chamber varied significantly between different tissue types and during the period of the investigation. Therefore, any method that does not correct for tissue optical properties and changes in autofluorescence leads to fluorescence quantification errors. We have shown that a ratiometric imaging method utilizing NIR autofluorescence detection can be applied and corrects for differences and changes in tissue optical properties and thickness. A limitation of our method is that for very early time points after the photosensitizer administration, a dependence on vessel size was found due to a mismatch in localization of marker fluorophore and background autofluorescence. Other than this limitation, the method we present shows a high sensitivity even for high photosensitizer concentrations.

Open Access This article is distributed under the terms of the Creative Commons Attribution Noncommercial License which permits any noncommercial use, distribution, and reproduction in any medium, provided the original author(s) and source are credited.

\section{References}

1. Lehr HA, Leunig M, Menger MD, Nolte D, Messmer K (1993) Dorsal skinfold chamber technique for intravital microscopy in nude mice. Am J Pathol 143(4):1055-1062

2. Clark ER, Kirby-Smith HT, Rex RO, Williams RG (1930) Recent modifications in the method of studying living cells and tissues in transparent chambers inserted in the rabbit's ear. Anat Rec $47: 187-211$

3. Algire GH (1943) An adaptation of the transparent chamber technique to the mouse. J Natl Cancer Inst 4:1-11

4. Branemark PL, Aspegren K, Breine U (1964) Microcirculatory studies in man by high-resolution vital microscopy. Angiology $15: 329-332$

5. Greenblatt M, Shubik P (1967) Hamster cheek pouch chamber. Cancer Bull 19:65-81

6. Arfors KE, Jonsson JA, McKenzie FN (1970) A titanium rabbit ear chamber: assembly, insertion and results. Microvasc Res 2:516-519

7. Hobbs JB, Chusilp S, Hua A, Kincaid-Smith P, McIver MA (1976) The pathogenesis of hypertensive vascular changes in the rat: microscopic and ultrastructural correlation in vivo. Clin Sci 51:71-75

8. Papenfuss HD, Gross JF, Intaglietta M, Treese FA (1979) A transparent access chamber for the rat dorsal skin fold. Microvasc Res 18:311-318

9. Kruijt B, de Bruijn HS, van der Ploeg-van den Heuvel A, Sterenborg HJCM, Robinson DJ (2006) Laser speckle imaging of dynamic changes in flow during photodynamic therapy. Laser Med Sci 21:208-212

10. van der Veen N, van Leengoed HLLM, Star WM (1994) In vivo fluorescence kinetics and photodynamic therapy using 5aminolaevulinic acid-induced porphyrin: increased damage after multiple irradiations. Br J Cancer 70:867-872

11. de Bruijn HS, Kruijt B, van der Ploeg-van den Heuvel A, Sterenborg HJCM, Robinson D (2007) Increase in protoporphyrin
IX after 5-aminolevulinic acid based photodynamic therapy is duet o local re-synthesis. Photochem Photobiol Sci 6:857-864

12. van Leengoed HLLM, van der Veen N, Versteeg AAC, Ouellet R, van Lier JE, Star WM (1993) In vivo fluorescence kinetics of phthalocyanines in a skin-fold observation chamber model: Role of central metal ion and degree of sulfonation. Photochem Photobiol 58(2):233-237

13. Cherry SR (2004) In vivo molecular and genomic imaging: new challenges for imaging physics. Phys Med Biol 49:R1348

14. Bogaards A, Sterenborg HJCM, Wilson BC (2007) In vivo quantification of fluorescent molecular markers in real-time: A review to evaluate the performance of five existing methods. Photodiagn Photodyn 4:170-178

15. Sinaasappel M, Sterenborg HJCM (1993) Quantification of the hematoporphyrin derivative by fluorescence measurement using dual-wavelength excitation and dual wavelength detection. Appl Optics 32(4):541-548

16. Bard MPL, Amelink A, Noordhoek Hegt V, Graveland WJ, Sterenborg HJCM, Hoogsteden HC, Aerts JGJV (2005) Measurement of hypoxia-related parameters in bronchial mucosa by use of optical spectroscopy. Am J Resp Cri Care Med 171:1179-1184

17. Amelink A, Kaspers OP, Sterenborg HJCN, van der Wal JE, Roodenburg JLN, Witjes MJH (2008) Non-invasive measurement of the morphology and physiology of oral mucosa by use of optical spectroscopy. Oral Oncol 44:65-71

18. Beauvoit B, Chance B (1998) Time-resolved spectroscopy of mitochondria, cells and tissue under normal and pathological conditions. Mol Cell Biochem 184:445-455

19. Profio A (1984) Laser excited fluorescence of hematoporphyrin derivative for diagnosis of cancer. IEEE J Quantum Elect QE20:1502-1507

20. Baumgartner R, Fisslinger H, Jocham D, Lenz H, Ruprecht L, Stepp H, Unsold E (1987) A fluorescence imaging device for endoscopic detection of early stage cancer-instrumental and experimental studies. Photochem Photobiol 46:759-763

21. Witjes MJH, Speelman OC, Nikkels PG, Nooren CAAM, Nautaj JM, van der Holt B, van Leengoed HLLM, Star WM, Roodenburg JLN (1996) In vivo fluorescence kinetics and localisation of aluminum phthalocyanine disulphonate in an autologous tumour model. Br J Cancer 73(5):573-580

22. Saarnak AE, Rodrigues T, Schwartz J, Moore AL, Gust D, van Gernert MJC, Sterenborg HJCM, Thomsen S (1998) Influence of tumour depth, blood absorption and autofluorescence on measurement of exogenous fluorophores in tissue. Lasers Med Sci 13:22-31

23. Reinhold HS, Blachiewicz B, van den Berg-Blok AE (1979) Reoxygenation of tumors in "sandwich" chambers. Eur J Cancer 15:481-489

24. Bogaards A, Sterenborg HJCM, Trachtenberg J, Wilson BC, Lilge L (2007) In vivo quantification of fluorescent molecular markers in real-time by ratio imaging for diagnostic screening and image-guided surgery. Lasers Surg Med 39:603-611

25. Kruijt B, de Bruijn HS, van der Ploeg-van den Heuvel A, de Bruin RWF, Sterenborg HJCM, Amelink A, Robinson DJ (2008) Monitoring ALA-induced PpIX photodynamic therapy in the rat esophagus using fluorescence and reflectance spectroscopy. Photochem Photobiol 84:1515-1527

26. de Veld DCG, Witjes MJH, van der Wal JE, Sterenborg HJCM, Roodenburg JLN (2005) The status of in vivo autofluorescence spectroscopy and imaging for oral oncology. Oral Oncol 41 (2): $117-131$

27. de Veld DCG, Skurichina M, Witjes MJH, Duin RPW, Sterenborg HJCM, Star WM, Roodenburg JLN (2003) Autofluorescence characteristics of healthy oral mucosa at different anatomical sites. Lasers Surg Med 32(5):367-376 
28. Triesscheijn M, Ruevekamp M, Out R, Van Berkel TJC, Schellens J, Baas P, Stewart FA (2007) The pharmacokinetic behavior of the photosensitizer meso-tetra-hydroxyphenyl-chlorin in mice and men. Cancer Chemoth Pharm 60:113-122

29. Jones HJ, Vernon DI, Brown SB (2003) Photodynamic therapy effect of m-THPC (Foscan) in vivo: correlation with pharmacokinetics. Brit J Cancer 89:398-404

30. Triesscheijn M, Ruevekamp M, Aalders M, Baas P, Stewart FA (2005) Outcome of mTHPC mediated therapy is primarily determined by the vascular response. Photochem Photobiol 81:1161-1167

31. Cramers P, Ruevekamp M, Oppelaar H, Dalesio O, Baas P, Stewart FA (2003) Foscan uptake and tissue distribution in relation to photodynamic efficacy. Brit J Cancer 88:283-290

32. Kruijt B, van der Ploeg-van den Heuvel A, de Bruijn HS, Sterenborg HJCM, Amelink A, Robinson DJ (2009) Monitoring interstitial $\mathrm{m}$-THPC-PDT in vivo using fluorescence and reflectance spectroscopy. Lasers Surg Med 41(9):653-664 